\title{
The BRIDGE Trial: What the Hospitalist Should Know
}

\author{
Bassel Nazha, MD, MPH ${ }^{1 \star}$, Alex C. Spyropoulos, MD²
}

${ }^{1}$ Department of Medicine, Staten Island University Hospital-Northwell Health, Staten Island, New York; ${ }^{2}$ Department of Medicine, Anticoagulation and Clinical Thrombosis Services, Hofstra-Northwell Health School of Medicine, Northwell Health at Lenox Hill Hospital, New York, New York.

Atrial fibrillation patients often require warfarin interruption for an invasive procedure or surgery. Heparin bridging therapy has been frequently used during warfarin interruption under the premise of providing a theoretical mitigation against thromboembolism that overweighs expected higher rates of bleeding. Up until recently, little definite clinical evidence was available to guide the hospitalist on optimal perioperative anticoagulant management. The landmark BRIDGE (Perioperative Bridging Anticoagulation in Patients with Atrial Fibrillation) trial provided high-quality evidence that a simple interruption of warfarin in the average atrial fibrillation patient undergoing an elective procedure or surgery is noninferior to bridging therapy for efficacy and superior to bridging therapy in preventing major bleeding. To guide the hospitalist, we propose a treatment algorithm based on these recent data. We review the literature that led to the trial and highlight its practice-changing implications as a proof of concept that calls to question the premise of heparin bridging therapy beyond the atrial fibrillation population. Journal of Hospital Medicine 2016;11:652-657. (C) 2016 Society of Hospital Medicine
In the United States, it is estimated that 2.7 to 6.1 million people have atrial fibrillation (AF). ${ }^{1}$ This number is projected to increase to 12.1 million in $2030 .^{2}$ Despite the advent of direct oral anticoagulants (DOAC), roughly half of patients with $\mathrm{AF}$ on anticoagulation are treated with vitamin $\mathrm{K}$ antagonists (VKA), warfarin being the most widely used. ${ }^{3}$

Every year at least 250,000 individuals will require anticoagulation interruption for an elective procedure. ${ }^{4}$ Clinicians, especially in hospitalized settings, are faced with the need to balance the risk of procedural bleeding with the potential for arterial thromboembolic (ATE) events. This is further complicated by warfarin's long half-life (36-60 hours). ${ }^{5}$ The slow weaning off and restoration of warfarin's anticoagulant effect expose patients, in theory, to a higher risk of ATE in the perioperative period. Heparin bridging therapy with unfractionated heparin (UFH) or low-molecular-weight heparin (LMWH) was believed to be a solution to provide continuous anticoagulant effect during temporary interruption of warfarin. Perioperative bridging therapy remains widely used by hospitalists, despite uncertainties about whether it meets its premise of conferring a clinically meaningful reduction of ATE's risk that overweighs the likely higher incidence of major bleeding associated with its use over a no-bridging strategy. Up until recently, no randomized clinical trials have

\footnotetext{
*Address for correspondence and reprint requests: Bassel Nazha, MD, MPH, Department of Medicine, Staten Island University Hospital-Northwell Health, 475 Seaview Avenue, Staten Island, NY 10305; Telephone: 718226-6205; Fax: 718-226-8695; E-mail: bnazha@northwell.edu

Additional Supporting Information may be found in the online version of this article.

Received: January 1, 2016; Revised: March 14, 2016; Accepted: March 24, 2016

2016 Society of Hospital Medicine DOI 10.1002/jhm.2594

Published online in Wiley Online Library (Wileyonlinelibrary.com).
}

evaluated the fundamental question of "should we bridge". The landmark BRIDGE (Perioperative Bridging Anticoagulation in Patients with Atrial Fibrillation) trial published in August 2015 greatly contributed to answering this question. ${ }^{6}$

In this article we perform a narrative review of the literature on the perioperative anticoagulation management of patients with AF on chronic warfarin needing an elective procedure or surgery that led to the BRIDGE trial. We also examine the most recent 9th Edition Guidelines from the American College of Chest Physicians (ACCP) on perioperative management of anticoagulation in this population. ${ }^{4}$ We then discuss in detail findings from the BRIDGE trial along with its implications for the hospitalist. Further, we suggest a practical treatment algorithm to the perioperative anticoagulation management of patients with AF on warfarin who are undergoing an elective procedure or surgery. We opt to focus on warfarin and to omit DOAC and antiplatelet therapies in our suggested practical approach. We lastly evaluate ongoing trials in this field.

\section{RECENT STUDIES ON HEPARIN BRIDGING IN ATRIAL FIBRILLATION USING CONTROL GROUPS}

In the last five years a body of evidence has progressively questioned the value of perioperative bridging therapy in preventing ATEs. The ORBIT-AF (Outcomes Registry for Better Informed Treatment of Atrial Fibrillation) study examined data on oral anticoagulation (OAC) interruption among 2200 patients in the United States. ${ }^{7}$ Patients who received bridging therapy accounted for $24 \%$ of interruptions and had a slightly higher $\mathrm{CHADS}_{2}$ score than non-bridged groups $(2.53$ vs $2.34, P=0.004)$. Overall, no significant differences in the rate of stroke or systemic embolism were detected between the bridged and nonbridged groups $(0.6 \%$ vs 
$0.3 \%, P=0.3)$. In multivariate analysis, bridging was associated with an odds ratio (OR) of 3.84 of major bleeding within 30 days $(P<0.0001)$, along with a higher 30-day composite incidence of myocardial infarction, stroke or systemic embolism, bleeding, hospitalization, or death (OR: 1.94, $P=0.0001$ ). The increased adverse events with bridging therapy were independent of the baseline OAC (warfarin or dabigatran). Although the study argued against the routine use of bridging in AF patients, the authors could not exclude the potential impact of measured $\left(\mathrm{CHADS}_{2}\right)$ and unmeasured confounding variables. ${ }^{7}$

The open-label RE-LY (Randomized Evaluation of Long Term Anticoagulant Therapy With Dabigatran Etexilate) trial compared dabigatran to warfarin in nonvalvular AF. Its dataset provided prospective information on 1424 warfarin interruptions for an elective procedure or surgery. The interruptions, of which $27.5 \%$ were treated with bridging therapy, were analyzed in a substudy of the trial. ${ }^{8}$ The $\mathrm{CHADS}_{2}$ or $\mathrm{CHA}_{2} \mathrm{DS}_{2}$-VASC scores were similar in the bridged and nonbridged warfarin groups. Relatively higher rates of major bleeding were observed in the bridged group $(6.8 \%$ vs $1.6 \%, P<0.001)$ with no statistically significant difference in stroke and systemic embolism $(0.5 \%$ vs $0.2 \%, P=0.32)$ compared to the nonbridged group. Paradoxically, bridging therapy was associated with a 6-fold increase in the risk of any thromboembolic event among patients on warfarin $(P$ $=0.007)$. As in the ORBIT-AF study, it was difficult to determine whether this increase was secondary to unmeasured confounding variables associated with higher baseline risk of ATE. ${ }^{8}$

The problem of unmeasured variables was common to the previous studies of perioperative bridging therapy. The heterogeneity of event definitions, bridging regimens, and per-protocol adherence rates were additional limitations to the studies' clinical implications, despite the consistency of a 3- to 4-fold increase in the major bleeding risk among bridged patients with no accompanying protection against ATE. From this perspective, the absence of high-quality data was the motivating force behind the BRIDGE trial.

\section{THE BRIDGE TRIAL}

The BRIDGE trial ${ }^{6}$ attempted to answer a simple yet fundamental question: in patients with AF on warfarin who need temporary interruption for an elective procedure or surgery, is perioperative heparin bridging necessary?

Adult patients ( $\geq 18$ years of age) were eligible for the study if they had chronic AF treated with warfarin for 3 months or more with a target International Normalized Ratio (INR) range of 2.0 to 3.0, $\mathrm{CHADS}_{2}$ score $\geq 1$, and were undergoing an elective invasive procedure or nonurgent surgery. The study excluded patients planned for a cardiac, intracranial, or intraspinal surgery. A history of stroke, ATE, or TIA in the preceding 3 months; a major bleed in the previous 6 weeks; or a mechanical heart valve precluded study participation. Further, those with a platelet count $<100,000 / \mathrm{mm}^{3}$ or creatinine clearance less than 30 $\mathrm{mL}$ per minute were also excluded.

Patients were randomly assigned to receive LMWH (dalteparin $100 \mathrm{IU} / \mathrm{kg}$ of body weight) or placebo subcutaneously twice daily in a double-blind fashion. In all patients, warfarin was withheld 5 days before the invasive procedure or elective surgery and restarted within 24 hours afterward. The bridging arm received therapeutic-dose LMWH starting 3 days before the procedure with matching placebo in the nonbridged arm. The last dose of LMWH or placebo was given around 24 hours before the procedure and then withheld. LMWH or placebo was restarted 12 to 24 hours after the procedure for defined low bleeding-risk procedures and 48 to 72 hours for high bleeding-risk procedures. The study drug was continued for 5 to 10 days and stopped when the INR was in the therapeutic range. The co-primary outcomes were ATE (stroke, TIA, or systemic embolism) and major bleeding using a standardized definition. These outcomes were assessed in the 30 days following the procedure.

Out of 1884 recruited patients in the United States and Canada, 934 patients were assigned to the bridging arm and 950 to the nonbridging arm. Study participants had a mean age of 71.7 years, a $\mathrm{CHADS}_{2}$ score of 2.3 , and 3 out of 4 were men. The 2 arms had similar baseline characteristics. Adherence to the study-drug protocol was high, with an $86.5 \%$ rate of adherence before the procedure to $96.5 \%$ after the procedure. At 30 days, the rate of ATE in the bridging group $(0.4 \%)$ was noninferior to the nonbridging one $(0.3 \%)(95 \%$ confidence interval $[\mathrm{CI}]:-0.6$ to $0.8 ; P$ value for noninferiority $=0.01$ ). The mean $\mathrm{CHADS}_{2}$ score in patients who sustained an ATE event was 2.6 (range, 1-4). The median time to an ATE event was 19.0 days (interquartile range [IQR], 6.0-23.0 days). The bridging group had a significantly higher rate of major bleeding compared to the nonbridging one (3.2\% vs $1.3 \%, P=0.005)$. The median time to a major bleeding event after a procedure was 7.0 days (IQR, 4.0-18.0 days). The 2 arms did not differ in their rates of venous thromboembolic (VTE) events and death in the study period. Yet, there was a significantly greater rate of minor bleeding in the bridging group $(20.9 \%$ vs $12.0 \%, P<0.001)$ and a trend toward more episodes of myocardial infarction in the bridging group as well $(1.6 \%$ vs $0.8 \%, P=0.10)$.

The BRIDGE trial was a proof of concept that the average AF patient may safely undergo commonly performed elective procedures or surgeries in which warfarin is simply withheld 5 days before and reinitiated within a day of the procedure without the need for periprocedural heparin bridging. Perioperative ATE rates, previously thought to be around $1 \%$, have been overestimated. The ATE rate was low in the 
TABLE 1. ACCP Risk Stratification of Perioperative Thromboembolism ${ }^{4}$

\begin{tabular}{|c|c|c|c|}
\hline Risk Category & Mechanical Heart Valve & Atrial Fibrillation & Venous Thromboembolism \\
\hline High & $\begin{array}{l}\text { Mitral valve prosthesis } \\
\text { Caged-ball or tiling-disc aortic valve prosthesis } \\
\text { Recent (<6 months) stroke or TIA }\end{array}$ & $\begin{array}{l}\text { CHADS }_{2} \text { score of } 5 \text { or } 6 \\
\text { Recent }(<3 \text { months) stroke or TIA } \\
\text { Rheumatic valvular heart disease }\end{array}$ & $\begin{array}{l}\text { Recent ( }<3 \text { month) VTE } \\
\text { Severe thrombophilia } \\
\text { Deficiency of protein C, protein S, or antithrombin } \\
\text { Antiphospholipid antibodies } \\
\text { Multiple thrombophilias }\end{array}$ \\
\hline Intermediate & $\begin{array}{l}\text { Bileaflet aortic valve prosthesis with a } \\
\text { major risk factor for stroke }\end{array}$ & $\mathrm{CHADS}_{2}$ score of 3 or 4 & $\begin{array}{l}\text { VTE within past 3-12 months } \\
\text { Nonsevere thrombophilia } \\
\text { Recurrent VTE } \\
\text { Active cancer }\end{array}$ \\
\hline Low & Bileaflet aortic valve prosthesis without a major risk factor for stroke & $\mathrm{CHADS}_{2}$ score of 0 to 2 with no prior stroke or TIA & VTE $>12$ months previous \\
\hline
\end{tabular}

ACCP: American College of Chest Physicians, TIA: Transient Ischemic Attack, VTE: Venous Thromboembolism.

BRIDGE trial $(\sim 0.4 \%)$, especially given a representative AF study population. The classical concern that warfarin interruption leads to a rebound hypercoagulable state was not supported by the trial.

The 9th Edition 2012 ACCP Guidelines on perioperative management of anticoagulation had suggested bridging in AF patients at high thrombotic risk and no bridging in the low risk group (Table 1). ${ }^{4}$ For patients at moderate risk, the ACCP Guidelines called for an individualized assessment of risk versus benefits of bridging, a recommendation that was not based on high-quality data. The BRIDGE trial findings are likely to change practice by providing level 1 evidence to forgo bridging in the vast majority of represented AF patients. For the hospitalist, this should greatly simplify periprocedural anticoagulant management for the AF patient on chronic warfarin in a hospitalized setting.

Limitations of the BRIDGE trial include the exclusion of surgeries that have an inherent high risk of postoperative thrombosis as well as bleeding, such as cardiac and vascular surgeries. Also, the trial had an underrepresentation of patients with a $\mathrm{CHADS}_{2}$ score of 5 or 6 and excluded those with a mechanical heart valve. Both of these groups carry a high risk of ATE. However, it would be expected that the increase in postprocedural bleed risk seen with therapeutic-dose bridging therapy in the BRIDGE trial would only be magnified in high bleeding-risk procedures, with either no effect on postoperative ATE risk reduction, or the potential to cause an increase in downstream ATE events by the withholding of anticoagulant therapy for a bleed event. The ongoing placebo-controlled PERIOP-2 trial (ClinicalTrials.gov no. NCT00432796) $)^{9}$ utilizes a strategy of dose adaptation of bridging therapy based on procedural bleeding risk, rather than a strategy of changing the timing of reinitiation of bridging therapy seen in the BRIDGE trial. Though the bridging protocol adapted in PERIOP-2 is used less often in clinical practice, the study is including patients with mechanical heart valves as well as following patients for a longer period of time compared to the BRIDGE trial (90 vs 30 postoperative days). This may elucidate the potential increase in downstream ATE events due to bleed events incurred by heparin bridging. The trial is planned to be completed in March 2017.

\section{PRACTICAL APPROACH TO PERIOPERATIVE MANAGEMENT OF WARFARIN ANTICOAGULATION IN ATRIAL FIBRILLATION}

In Figure 1 we suggest a practical 3-step framework for the perioperative anticoagulation management of patients on chronic warfarin for AF. First, if the planned invasive procedure or surgery falls under the minimal bleeding-risk group in Table 2, we propose continuing warfarin in the perioperative period. Notably, implantation of a pacemaker or cardioverterdefibrillator device is included in this group based on recently completed randomized trials in this patient group. In fact, the BRUISE CONTROL trial showed a markedly reduced incidence of device-pocket hematoma when warfarin was continued in the perioperative period as compared to its temporary interruption and use of bridging $(3.5 \%$ vs $16 \%, P<0.001)$. Other surgical complications including ATE events were similar in the 2 groups. ${ }^{10}$ The COMPARE trial demonstrated that warfarin can also be continued in the periprocedural period in patients undergoing catheter ablation of AF. Warfarin's continuation among 1584 AF patients who had this procedure was associated with significantly fewer thromboembolic events $(0.25 \%$ vs $4.9 \%$, $P<0.001)$ and minor bleeding complications $(4.1 \%$ vs $22 \%, P<0.001)$ compared to its temporary interruption and use of bridging. ${ }^{11}$ We recognize that the clinical distinction between minimal and low bleeding risk can be difficult, yet the former is increasingly recognized as a group in which anticoagulation can be safely continued in the perioperative period. ${ }^{12}$

Second, if the decision was made to hold warfarin, the next step is to estimate the patient's perioperative 


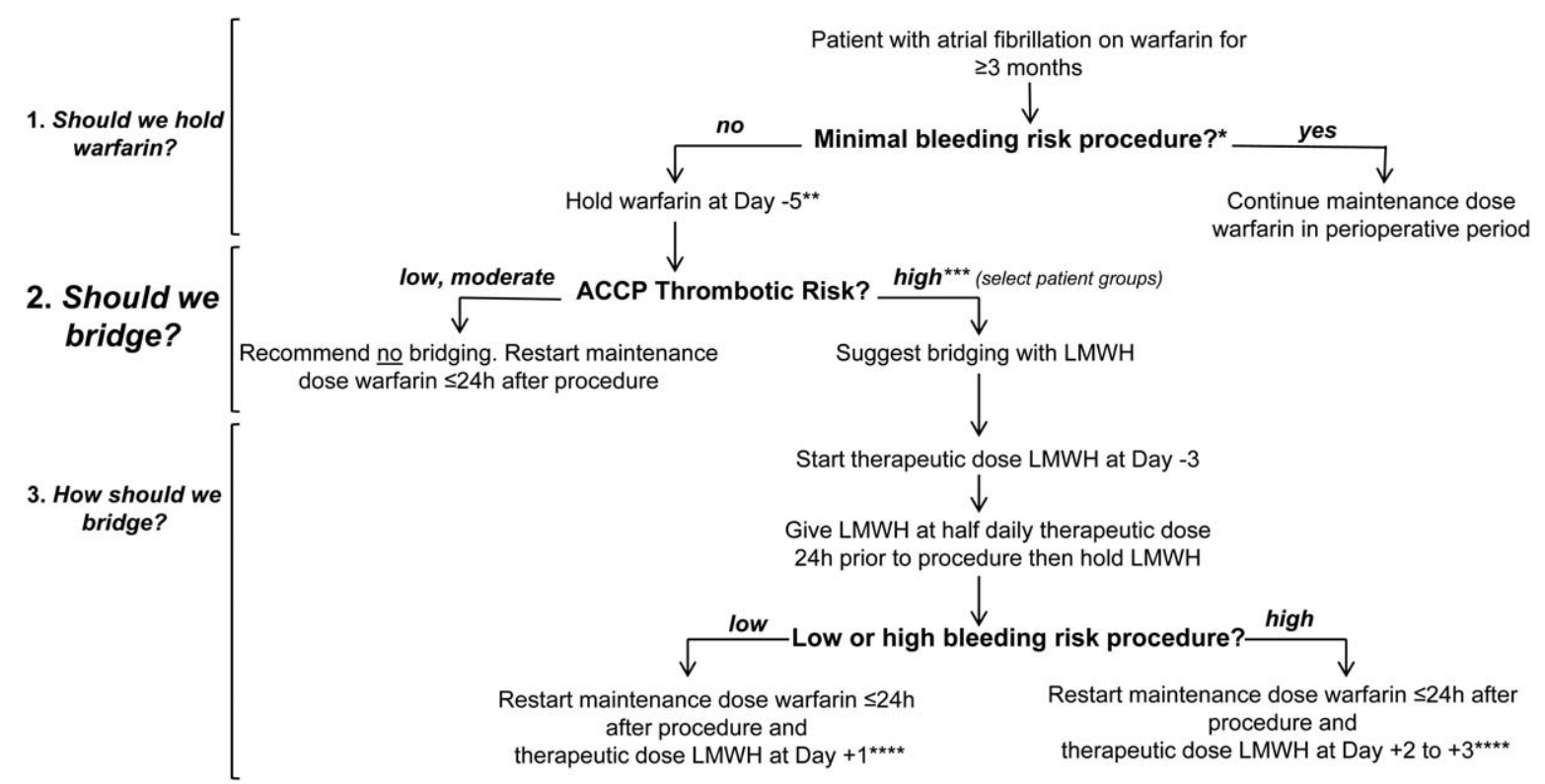

FIG. 1. Suggested periprocedural management of warfarin anticoagulation in chronic atrial fibrillation based on the most recent clinical evidence. *Includes pacemaker and cardioverter-defibrillator device implantation, and catheter ablation of atrial fibrillation as level 1 evidence indicates that they can be done without warfarin interruption (Table 2). ${ }^{* \star}$ For patients with International Normalized Ratio (INR) target range of 2.5 to 3.5 and elderly patients, we suggest holding warfarin on day 6 (the procedure being on day 0). ${ }^{\star \star \star}$ Especially valvular atrial fibrillation associated with (1) a mechanical heart valve, (2) a recent stroke or transient ischemic attack, or (3) severe rheumatic heart disease. There were few patients in BRIDGE with a $\mathrm{CHADS}_{2}$ score of 5 or $6 .{ }^{* \star \star \star}$ Therapeutic-dose low-molecular-weight heparin (LMWH) may be stopped once INR $\geq 2$.

thrombotic risk based on the 9th Edition ACCP Guidelines shown in Table 1. Whereas patients may have additional comorbidities, a theoretical framework for an individual patient's ATE risk stratification as seen in the ACCP Guidelines is determined by the $\mathrm{CHADS}_{2}$ score, a history of rheumatic heart disease, and a recent ATE event (within 3 months). In the low ATE risk group, recommendations from the ACCP, ${ }^{4}$ the American Heart Association, and the American College of Cardiology ${ }^{13}$ are in agreement against the use of perioperative bridging. Level 1 evidence from the BRIDGE trial now supports that bridging may be forgone in patients in the moderate ATE risk group and likely many patients in the high ATE risk group (although patients with a $\mathrm{CHADS}_{2}$ score of 5 and 6 were under-represented in the BRIDGE trial). In certain high ATE risk patient groups with AF, especially those with a recent ATE event, mechanical heart valves, or severe rheumatic heart disease, it may be prudent to bridge those patients with UFH/LMWH.

Third, assuming adequate hemostasis is achieved after the procedure, warfarin can be restarted within 24 hours at its usual maintenance dose regardless of bridging. For patients among whom bridging is chosen, we suggest that the timing of resumption of LMWH bridging be based on the procedural risk of bleeding (Table 2): 1-day postprocedurally in the low bleeding-risk groups or 2 to 3 days postprocedurally in the high bleeding-risk groups. For the latter group, a stepwise use of prophylactic-dose LMWH, especially after a

TABLE 2. Suggested Stratification of Procedural Bleeding Risk

\begin{tabular}{lll}
\hline Minimal Bleeding-Risk Procedures & \multicolumn{1}{c}{ Low Bleeding-Risk Procedures } & High Bleeding-Risk Procedures \\
\hline $\begin{array}{l}\text { Implantation of pacemaker or cardioverter-defibrillator device; } \\
\text { catheter ablation of atrial fibrillation* }\end{array}$ & Coronary angiography & Cardiac, intracranial, or spinal surgery; any major procedure lasting \\
$\begin{array}{l}\text { Minor cutaneous excision (actinic keratosis, premalignant/malignant } \\
\text { skin nevi, basal and squamous cell skin carcinoma) }\end{array}$ & Cutaneous or lymph node biopsy & Major surgery with extensive tissue resection; \\
Cataract surgery & cancer surgery \\
& Arthroscopy; & Major orthopedic surgery \\
Minor dental procedure (cleaning, filling, extraction, & surgery of hand, foot, or shoulder \\
endodontic, prosthetic) & $\begin{array}{c}\text { Endoscopy/colonoscopy } \pm \text { biopsy, laparoscopic cholecystectomy, } \\
\text { hemorrhoidal surgery, abdominal hernia repair }\end{array}$ & $\begin{array}{c}\text { Liver or spleen surgery, bowel resection, colonic polyp resection, } \\
\text { percutaneous endoscopic gastrotomy placement, endoscopic } \\
\end{array}$ \\
& Bronchosopy & retrograde cholangiopancreatography \\
& & Nephrectomy, kidney biopsy, transurethral prostate resection, bladder \\
& resection, or tumor ablation
\end{tabular}

*Level 1 evidence supports continuation of oral anticoagulation in perioperative period, as this approach results in significantly fewer pocket hematomas compared to temporary oral anticoagulation interruption and use of bridging therapy. ${ }^{10,11}$ 
major surgery for the prevention of VTE, may be resumed earlier at the discretion of the surgeon or interventionist. For both groups, therapeutic-dose LMWH may be stopped once the INR is $\geq 2$.

A number of challenges are associated with the proposed framework. Real-world data show that nonindicated OAC interruptions and bridging are commonplace. This may defer the hospitalist's readiness to change practice. ${ }^{7}$ Although the $\mathrm{CHADS}_{2} / \mathrm{CHA}_{2} \mathrm{DS}_{2}$-VASc scores are widely used to estimate the perioperative ATE risk, there is scant evidence from validation studies, ${ }^{14,15}$ whereas the $\mathrm{CHADS}_{2}$ score has been used in guideline recommendations. ${ }^{4}$ Also, as previously discussed, this framework excludes patients with a recent stroke or a mechanical heart valve, patients on warfarin for VTE, and patients on DOACs.

\section{RETHINKING HEPARIN BRIDGING THERAPY IN NON-ATRIAL FIBRILLATION PATIENT GROUPS}

There is now mounting recent evidence from over 12,000 patients that any heparin-based bridging strategy does not reduce the risk of ATE events but confers an over 2- to 3 -fold increased risk of major bleeding. ${ }^{16}$ Thus, in our view, the BRIDGE trial was a proof of concept that calls to question the premise of heparin bridging therapy in preventing ATE beyond the AF population. Retrospective studies provide evidence of the lack of treatment effect with heparin bridging even in perceived high thromboembolic risk populations, including those with mechanical heart valves and VTE (2 patient groups for whom there are currently no level 1 data on perioperative management of anticoagulation and bridging therapy).

In their systematic review and meta-analysis, Siegal et al. evaluated periprocedural rates of bleeding and thromboembolic events in more than 12,000 patients on VKA based on whether they were bridged with control groups. ${ }^{16}$ Thirty out of 34 studies reported the indication for anticoagulation, with AF being the most common $(44 \%)$. Bridging was associated with an OR of 5.4 for overall bleeding (95\% CI: 3.0 to 9.7) and an OR of 3.6 for major bleeding (95\% CI: 1.5 to 8.5). ATE and VTE events were rare, with no statistically significant differences between the bridged $(0.9 \%)$ and nonbridged patients $(0.6 \%)$ (OR: $0.8,95 \%$ CI: 0.42 to 1.54). The authors suggested that bridging might better be reserved to patients who are at high risk of thromboembolism. Nonetheless, the implications of the findings were limited by the poor quality of included studies and their heterogeneity in reporting outcomes, especially bleeding events. ${ }^{16}$

In a retrospective cohort study of 1777 patients who underwent mechanical heart valve replacement $(56 \%$ aortic, 34\% mitral, 9\% combined aortic and mitral), 923 patients who received therapeutic-dose bridging therapy in the immediate post-valve implantation period had a 2.5 to 3 times more major bleeding $(5.4 \%$ vs $1.9 \%, P=0.001$ ) and a longer hospital stay compared to those who received prophylactic-dose bridging anticoagulation. The two groups had comparable thromboembolic complications at 30 days $(\approx 2 \%, P=$ 0.81). ${ }^{17}$ Another study retrospectively analyzed data from 1178 patients on warfarin for prevention of secondary VTE who had anticoagulation interruption for an invasive procedure or surgery. About onethird received bridging therapy, the majority with therapeutic-dose LMWH. Of the bridged patients, $2.7 \%$ had a clinically relevant bleeding at 30 days compared to $0.2 \%$ in the nonbridged groups $(P=0.01)$. The incidence of a recurrent VTE was low across all thrombotic risk groups, with no differences between bridged and nonbridged patients $(0.0 \%$ vs $0.2 \%, P=$ $0.56) .^{18}$

There are a number of factors as to why heparin bridging appears ineffective in preventing periprocedural ATE events. It is possible that rebound hypercoagulability and a postoperative thrombotic state have been overestimated. Older analyses supporting postoperative ATE rates of $\sim 1.6 \%$ to $4.0 \%$ and a 10 -fold increased risk of ATE by major surgery are not supported by recent perioperative anticoagulant studies with control arms, including the BRIDGE trial, where the ATE event rate was closer to $0.5 \%$ to $1.0 \% .^{6-8,19}$ The mechanisms of perioperative ATE may be more related to other factors than anticoagulant-related factors, such as the vascular milieu, ${ }^{14}$ alterations in blood pressure, ${ }^{20}$ improvements in surgical and anesthetic techniques (including increasing use of neuraxial anesthesia), ${ }^{21}$ and earlier patient mobilization. Indeed, the occurrence of ATE events in the BRIDGE trial did not appear to be influenced by a patient's underlying $\mathrm{CHADS}_{2}$ score (mean $\mathrm{CHADS}_{2}$ score of 2.6). There is a growing body of evidence that suggests perioperative heparin bridging has the opposite effect to that assumed by its use: there are trends toward an increase in postoperative ATE events in patients who receive bridging therapy. ${ }^{8}$

In the BRIDGE trial, there was a trend toward an increase in myocardial infarction in the bridging arm. This can be explained by a number of factors, but the most obvious includes an increase in bleeding events as may be expected by the use of therapeutic-dose heparin bridging over a no-bridging approach, which then predisposes a patient to downstream ATE events after withholding of anticoagulant therapy. The median time to a major bleed in BRIDGE was 7 days, whereas the mean time to an ATE event was 19 days, suggesting that bleeding is front-loaded and that withholding of anticoagulant therapy after a bleed event may potentially place a patient at risk for later ATE events. This is consistent with an earlier single-arm prospective cohort study of 224 high ATE risk patients on warfarin who were treated with perioperative LMWH bridging therapy. Among patients who had a thromboembolic event in the 90 postoperative days, $75 \%$ (6 out of 8 ) had their 
warfarin therapy withdrawn or deferred because of bleeding. ${ }^{22}$ Last, if prophylactic doses of heparin were used as bridging therapy, there is no evidence that this would be protective of ATE events, which is the premise of using heparin bridging. Both of these concepts will be assessed when results of the PERIOP-2 trial are made available.

An emerging body of evidence suggests an unfavorable risk versus benefit balance of heparin bridging, regardless of the underlying thrombotic risk. Overall, if bridging therapy is effective in protecting against ATE (which has yet to be demonstrated), recent studies show that its number needed to treat (NNT) would be very large and far larger than its number needed to harm (NNH). If more patients undergoing high bleeding-risk procedures were included in the BRIDGE trial, these effects of unfavorable NNT to $\mathrm{NNH}$ would be magnified. While awaiting more definite answers from future trials, we believe clinicians should be critical of heparin bridging. We also suggest that they reserve it for patients who are at a significantly high risk of ATE complications until uncertainties around its use are clarified.

\section{CONCLUSION}

The BRIDGE trial provided high-quality evidence that routine perioperative heparin bridging of patients on chronic warfarin for AF needing an elective procedure or surgery is both unnecessary and harmful. The trial is practice changing for patients with $\mathrm{AF}$, and its results will likely be implemented in future international guidelines on the topic, including those of the ACCP. The hospitalist should be aware that the current large body of evidence points to more harm than benefit associated with heparin bridging in preventing ATE for any patient group, including those at high risk of ATE. Ongoing and future trials may clarify the role of heparin bridging-if any-in patients on chronic warfarin at high risk of ATE, including those with mechanical heart valves.

Disclosures: Alex C. Spyropoulos, MD, has served as a consultant for Bayer, Boehringer Ingelheim, Daiichi Sankyo, and Janssen. He also has served on advisory committees for Bristol-Myers Squibb and Pfizer.

\section{References}

1. Centers for Disease Control and Prevention. Atrial fibrillation fact sheet. Available at: http://www.cdc.gov/dhdsp/data_statistics/fact sheets/fs_atrial_fibrillation.htm. Updated August 13, 2015. Accessed November 22, 2015 .

2. Colilla S, Crow A, Petkun W, Singer DE, Simon T, Liu X. Estimates of current and future incidence and prevalence of atrial fibrillation in the U.S. adult population. Am J Cardiol. 2013;112(8):1142-1147.
3. Barnes GD, Lucas E, Alexander GC, Goldberger ZD. National trends in ambulatory oral anticoagulant use. Am J Med. 2015;128(12):13001305.e2.

4. Douketis JD, Spyropoulos AC, Spencer FA, et al. Perioperative management of antithrombotic therapy: Antithrombotic Therapy and Prevention of Thrombosis, 9th ed: American College of Chest Physicians evidence-based clinical practice guidelines. Chest. 2012;141(2 suppl): e326S-e350S.

5. Ansell J, Hirsh J, Hylek E, et al. Pharmacology and management of the vitamin K antagonists: American College of Chest Physicians evidence-based clinical practice guidelines (8th edition). Chest. 2008; 133(6 suppl):160S-198S.

6. Douketis JD, Spyropoulos AC, Kaatz S, et al. Perioperative Bridging Anticoagulation in Patients with Atrial Fibrillation. N Engl J Med. 2015;373(9):823-833.

7. Steinberg BA, Peterson ED, Kim S, et al. Use and outcomes associated with bridging during anticoagulation interruptions in patients with atrial fibrillation: findings from the Outcomes Registry for Better Informed Treatment of Atrial Fibrillation (ORBIT-AF). Circulation. 2015;131(5):488-494.

8. Douketis JD, Healey JS, Brueckmann M, et al. Perioperative bridging anticoagulation during dabigatran or warfarin interruption among patients who had an elective surgery or procedure. Substudy of the RE-LY trial. Thromb Haemost. 2015;113(3):625-632.

9. PERIOP 2-A Safety and Effectiveness Study of LMWH Bridging Therapy Versus Placebo Bridging Therapy for Patients on Long Term Warfarin and Require Temporary Interruption of Their Warfarin. ClinicalTrials.gov. Available at: https://clinicaltrials.gov/ct2/show/ NCT00432796. Accessed December 9, 2015.

10. Birnie DH, Healey JS, Wells GA, et al. Pacemaker or defibrillator surgery without interruption of anticoagulation. N Engl J Med. 2013; 368(22):2084-2093.

11. Di Biase L, Burkhardt JD, Santangeli P, et al. Periprocedural stroke and bleeding complications in patients undergoing catheter ablation of atrial fibrillation with different anticoagulation management: results from the Role of Coumadin in Preventing Thromboembolism in Atrial Fibrillation (AF) Patients Undergoing Catheter Ablation (COMPARE) randomized trial. Circulation. 2014;129(25):26382644.

12. Bajkin BV, Vujkov SB, Milekic BR, Vuckovic BA. Risk factors for bleeding after oral surgery in patients who continued using oral anticoagulant therapy. J Am Dent Assoc. 2015;146(6):375-381.

13. Fleisher LA, Fleischmann KE, Auerbach AD, et al. 2014 ACC/AHA guideline on perioperative cardiovascular evaluation and management of patients undergoing noncardiac surgery: executive summary: a report of the American College of Cardiology/American Heart Association Task Force on Practice Guidelines. Circulation. 2014;130(24): 2215-2245.

14. Kaatz S, Douketis JD, Zhou H, Gage BF, White RH. Risk of stroke after surgery in patients with and without chronic atrial fibrillation. J Thromb Haemost. 2010;8(5):884-890.

15. Daniels PR. Peri-procedural management of patients taking oral anticoagulants. BMJ. 2015;351:h2391.

16. Siegal D, Yudin J, Kaatz S, Douketis JD, Lim W, Spyropoulos AC. Periprocedural heparin bridging in patients receiving vitamin $\mathrm{K}$ antagonists: systematic review and meta-analysis of bleeding and thromboembolic rates. Circulation. 2012;126(13):1630-1639.

17. Mathew JG, Spyropoulos AC, Yusuf A, et al. Efficacy and safety of early parenteral anticoagulation as a bridge to warfarin after mechanical valve replacement. Thromb Haemost. 2014;112(6):1120-1128.

18. Clark NP, Witt DM, Davies LE, et al. Bleeding, recurrent venous thromboembolism, and mortality risks during warfarin interruption for invasive procedures. JAMA Intern Med. 2015;175(7):1163-1168.

19. Dunn AS, Turpie AG. Perioperative management of patients receiving oral anticoagulants: a systematic review. Arch Intern Med. 2003; 163(8):901-908.

20. Cheung CC, Martyn A, Campbell N, et al. Predictors of intraoperative hypotension and bradycardia. Am J Med. 2015;128(5):532-538.

21. Selim M. Perioperative stroke. N Engl J Med. 2007;356(7):706-713.

22. Kovacs MJ, Kearon C, Rodger M, et al. Single-arm study of bridging therapy with low-molecular-weight heparin for patients at risk of arterial embolism who require temporary interruption of warfarin. Circulation. 2004;110(12):1658-1663. 